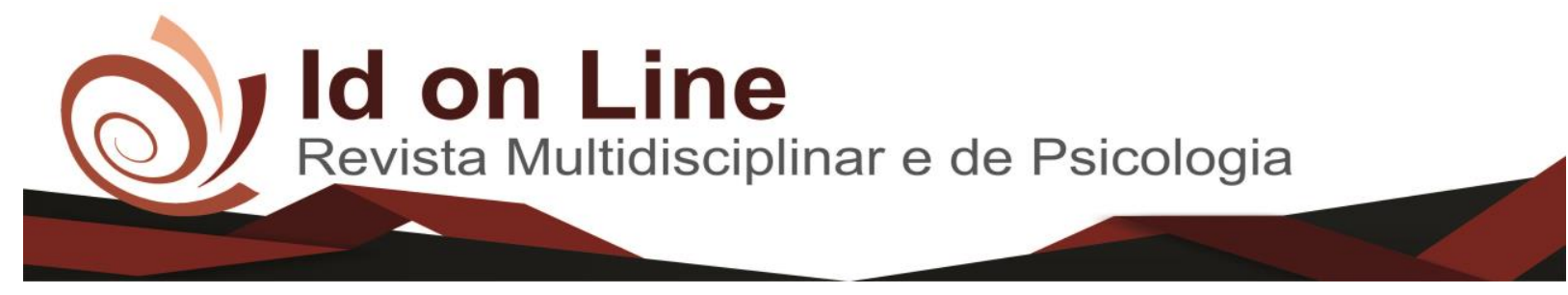

\title{
Perfil dos Pacientes portadores de Lúpus Eritematoso Sistêmico atendidos pelo Componente Especializado da Assistência Farmacêutica em uma Regional de Saúde
}

\author{
Diane de Matos Pradol; Bruno Andrade Amaral²; Stênio Fernando Pimentel Duarte; \\ Ademir Evangelista do Vale ${ }^{4}$; Matheus Lemos Silva ${ }^{5}$; Arlene Ribeiro Rocha ${ }^{6}$; Vitória Magalhães Sousa ${ }^{7}$.
}

\begin{abstract}
Resumo: O Lúpus Eritematoso Sistêmico (LES) é uma doença de caráter autoimune e crônico inflamatório, que possui etiologia multissistêmica e afeta principalmente as mulheres, em diversos órgãos, podendo estar ligada a fatores ambientais, hormonais e infecciosos. A doença é diagnosticada, por meio do auxílio de exames laboratoriais, a anamnese eficaz e correlação com critérios diagnósticos específicos, a fim de se iniciar o tratamento. Este artigo tem como objetivo descrever o perfil sociodemográfico e clínico dos pacientes portadores de LES atendidos pelo Componente Especializado da Assistência Farmacêutica em uma Regional de Saúde, observando o perfil sociodemográfico da população estudada, os exames laboratoriais que favorecem a observação da efetividade e segurança dos tratamentos instituídos e observação da correlação com os fatores de pré-disposição. Foi realizado estudo no NRS entre Agosto e Setembro de 2017, por meio de coleta de dados em prontuários, através do Kobocollect, sendo analisados posteriormente no software aplicativo SPSS. A maioria dos pacientes era do sexo feminino (95,5\%), pardos (58\%), faixa etária média de 41,08 anos, procedentes de Vitória da Conquista-BA. As alterações mais frequentes observadas foram: distúrbio hematológico $21 \%$ ( $\mathrm{n}=58$ ), hepático $19 \%(\mathrm{n}=35)$ e renal $16 \%(\mathrm{n}=16)$, sendo que os medicamentos mais frequentemente associados a esses distúrbios, foram a Azatioprina e Hidroxicloroquina. Na população em questão, os pacientes apresentaram um perfil condizente com o esperado pela literatura, sendo na sua maioria do sexo feminino, pardos, adultos jovens, com IMC normal, com presença de anormalidades hematológicas, hepáticas e renais.
\end{abstract}

Palavras-chave: Lúpus eritematoso sistêmico. Perfil epidemiológico. Efetividade de tratamento. Toxicidade. Assistência Farmacêutica.

\section{Perfil dos Pacientes portadores de Lúpus Eritematoso Sistêmico atendidos pelo Componente Especializado da Assistência Farmacêutica em uma Regional de Saúde}

\begin{abstract}
Systemic Lupus Erythematosus (SLE) is an autoimmune and chronic inflammatory disease, which has a multisystemic etiology and affects mainly women, in several organs, and may be linked to environmental, hormonal and infectious factors. The disease is diagnosed, through the aid of laboratory tests, the effective anamnesis and correlation with specific diagnostic criteria, in order to start the treatment. This article aims to
\end{abstract}

\footnotetext{
1 Diane de Matos Prado, graduanda em Farmácia pela Faculdade Independente do Nordeste - FAINOR. E-mail: dianematos@hotmail.com. Vitória da Conquista, Bahia, Brasil.

2 Bruno Andrade Amaral, farmacêutico graduado pela Universidade Federal da Bahia, mestrando no Programa de Pós-Graduação em Assistência Farmacêutica em rede (UFRGS/UFBA) e docente da Faculdade Independente do Nordeste - FAINOR. E-mail: brunofarmaceutico@ hotmail.com. Vitória da Conquista, Bahia, Brasil.

${ }^{3}$ Stênio Fernando Pimentel Duarte, doutor e mestre em Fisiopatologia Clínica e Experimental pela Universidade do Estado do Rio de Janeiro (UERJ). Graduado em Licenciatura Plena em Ciências Biológicas pela UERJ e docente da Faculdade Independente do Nordeste - FAINOR. E-mail: steniofernando@gmail.com.Vitória da Conquista, Bahia, Brasil.

${ }^{4}$ Ademir Evangelista do Vale, farmacêutico com mestrado e doutorado em Química pela Universidade Federal da Bahia -UFBA. Email: advale@gmail.com. Vitória da Conquista, Bahia, Brasil.

${ }^{5}$ Matheus Lemos Silva, graduando em nutrição, na Faculdade de Tecnologia e Ciências - FTC. Email: Matheus.nutri3@gmail.com. Vitória da Conquista, Bahia, Brasil.

6 Arlene Ribeiro Rocha, graduanda em Farmácia pela Faculdade Independente do Nordeste - FAINOR. E-mail: arleneribeirofarm@outlook.com. Vitória da Conquista, Bahia, Brasil.

7 Vitória Magalhães Sousa, graduanda em Farmácia pela Faculdade Independente do Nordeste - FAINOR. E-mail: vitooriamagalhaes@gmail.com. Vitória da Conquista, Bahia, Brasil.
} 
describe the sociodemographic and clinical profile of patients with SLE treated by the Specialized Component of Pharmaceutical Assistance in a Health Region, observing the sociodemographic profile of the population studied, laboratory tests that favor the observation of the effectiveness and safety of the treatments and the correlation with the pre-disposition factors. A study was carried out in the NRS between August and September 2017, through the collection of data in medical records, through Kobocollect, and analyzed later in the SPSS application software. The majority of the patients were female (95.5\%), brown (58\%), and average age of 41.08 years, from Vitória da Conquista-BA. The most frequent alterations observed were: hematologic disorder $21 \%(\mathrm{n}=58)$, hepatic $19 \%(\mathrm{n}$ $=35)$ and renal $16 \%(\mathrm{n}=16)$; the most frequent drugs associated with these disorders were azathioprine and Hydroxychloroquine. In the population in question, the patients presented a profile consistent with that expected in the literature, being mostly female, brown, young adults, with normal BMI, with hematological, hepatic and renal abnormalities.

Key words: Systemic lupus erythematosus. Epidemiological profile. Effectiveness of treatment. Toxicity. Pharmaceutical care.

\section{Introdução}

O sistema imunológico é um componente existente em todo indivíduo, que é responsável por produzir resposta imune contra qualquer corpo estranho (imunógeno) no organismo, onde atua eliminando de forma eficaz tal substância prejudicial (VIANNA; SIMÕES; INFORZATO, 2010).

Logo, a função fisiológica do sistema imunológico é defender o organismo contra qualquer elemento desconhecido, como por exemplo, micro-organismos infecciosos (ABBAS; LICHTMAN; PILLAI, 2008). Porém, pode haver um lapso nesse sistema, ocasionando ataque contra o próprio organismo, devido a falha relacionada a auto tolerância imunológica (VIANNA; SIMÕES; INFORZATO, 2010).

Essa falha, ocorre devido a um desequilíbrio do sistema imunológico, que resulta em alteração da resposta imune normal, seguida de produção de autoanticorpo, que age agredindo as células e tecidos do corpo, gerando a chamada doença autoimune inflamatória sistêmica. Estão incluídas nesse seguimento patológico de falha imunológica, doenças como a artrite reumatóide, o lúpus eritematoso sistêmico (LES), a dermatomiosite, a polimiosite, a esclerose sistêmica, as vasculites e a síndrome de Sjögren (GALINDO; VEIGA, 2010).

O Lúpus Eritematoso Sistêmico (LES) é uma doença autoimune, que leva à produção de autoanticorpos, com grau de especificidade elevada a estruturas do próprio organismo. Essa doença tem caráter inflamatório e crônico e pode estar relacionada a fatores genéticos, ambientais e hormonais. Pode apresentar períodos de exacerbação e remissão, sendo que a sua 
forma clínica é complexa, podendo comprometer várias regiões do corpo, como os rins, as articulações, as membranas serosas, a pele e de forma mais típica e frequente, os rins (SOUSA; SILVA; PARTATA, 2011).

Segundo Sato e colaboradores (2002), o LES tem maior incidência em mulheres do que em homens, numa proporção em torno de 9:1, podendo ocorrer em todas as raças, e em mulheres especialmente jovens. Os sinais e sintomas do LES mais comuns são fadiga, febre, emagrecimento, perda de apetite, fotossensibilidade, lesões na pele e articulações. Pode haver ainda acometimento de outros órgãos como o rim, o coração ou o sistema nervoso central (cérebro).

Devido ao polimorfismo clínico, se torna mais difícil reconhecer e diferenciar o quadro do paciente, fazendo com que haja um diagnóstico tardio, até então, ausente de terapêutica adequada, levando, portanto, a uma maior evolução da doença no paciente (AYACHE; COSTA, 2005).

O diagnóstico do Lúpus Eritematoso Sistêmico se baseia numa junção de fatores inerentes ao paciente, como manifestações clínicas características e de auto anticorpos, cautelosamente observados (HOCHBERG, 1997). Além dos achados laboratoriais característicos da doença, com a finalidade de ratificar o diagnóstico, foi criado em 1971 e publicados em 1982, critérios de classificação diagnóstica para LES pelo American College of Rheumatology (ACR), que mais tarde, em 1997, foram revisados por um comitê, a fim de eliminar o critério de célula LE e alterar o critério imunológico para incluir anticorpos anticardiolipina. Esses critérios por sua vez, foram submetidos à revisão e posteriormente autenticados pelo grupo Internacional de Colaboração Clínica em Lúpus Sistêmico (Systemic Lupus International Collaborating Clinics - SLICC), por meio de onze parâmetros itens clínicos e seis parâmetros imunológicos, sendo que é necessário que o paciente apresente quatro itens (entre eles, um item clínico e um item imunológico) ou nefrite lúpica comprovada por biópsia compatível com LES (autoanticorpos lúpicos presente) (PETRI et al., 2012).

Após o paciente ter a confirmação da doença, imediatamente deve se iniciar o tratamento, onde o paciente será orientado prática de melhores hábitos de vida, por meio de uma alimentação adequada, prática de exercício físico regular, não ingestão de bebida alcoólica e/ou uso do cigarro, proteção contra os raios UVA e UVA, e uso de tratamento medicamentoso adequado para sua condição clínica (SATO, 2002).

Para ter acesso ao tratamento medicamentoso pelo SUS, a Assistência farmacêutica (AF), se organiza através dos componentes da AF, e de acordo com a Portaria MS 1554/2013 
o Componente Especializado da Assistência Farmacêutica (CEAF) busca em nível ambulatorial, a garantia da integralidade da farmacoterapia ao paciente, por meio de linhas de cuidado para doenças contempladas, definidas em Protocolos Clínicos e Diretrizes Terapêuticas, de modo que estes pacientes serão orientados quanto a patologia, o tratamento e o risco-benefício do mesmo, sendo assim submetido a monitoramento do tratamento estabelecido, visando melhores resultados (BRASIL, 2013).

Assim, o objetivo deste estudo foi descrever o perfil sociodemográfico e clínico dos pacientes portadores de LES atendidos pelo CEAF em uma Regional de Saúde, verificando os resultados de exames laboratoriais marcadores do perfil de efetividade e segurança dos tratamentos instituídos, fazendo assim a correlação entre os fatores preditores (sexo, idade, associação dos medicamentos utilizados para o tratamento) e a efetividade e segurança da farmacoterapia instituída.

\section{Método}

O estudo possui delineamento observacional, transversal e foi desenvolvido em pacientes com LES atendidos no Núcleo Regional de Saúde - NRS de Vitória da Conquista, por meio do Componente Especializado da Assistência Farmacêutica, no período de Agosto à Setembro de 2017.

Foi obtida a aprovação do Comitê de Ética em Pesquisa da Faculdade Independente do Nordeste - FAINOR, sob o CAAE nº 69977317.0.0000.5578, sendo aprovada a solicitação de dispensa de TCLE, visto que não houve contato com os pacientes, apenas com os prontuários, de modo que os dados dos mesmos foram mantidos em sigilo.

A coleta de dados ocorreu por meio de revisão de prontuários, fazendo uma análise sociodemográfica e clínica de pacientes com CID-10: M32.1 e M32.8. Foram coletados dados pertinentes à pesquisa, como: identificação do paciente, data de nascimento, raça, idade, gênero, peso, altura, diagnóstico médico, tratamento medicamentoso e exames laboratoriais trimestrais.

A raça, sexo, peso e altura dos pacientes foram colhidos no Laudo para Solicitação, Avaliação e Autorização de Medicamentos (LME). Na prescrição e no Recibo de medicamentos (RME), foi possível coletar o tratamento medicamentoso utilizado bem como sua dispensação. A coleta dos exames foi feita a partir da análise dos exames entregue trimestralmente pelo paciente para monitoramento, onde foram analisadas as Hemácias, Leucócitos, Plaquetas, 
Transaminase glutâmico-oxalacética (TGO), Transaminase glutâmico-pirúvica (TGP), Fosfatase Alcalina, Creatinina, Uréia, velocidade de hemossedimentação (VHS) e a Proteína C reativa (PCR).

Os dados foram coletados e armazenados com o auxílio do aplicativo Kobocollect@, para posterior análise através do software IBM® SPSS Statistics 24.0 com níveis de confiança de 5\%, onde os resultados foram submetidos à análise Chi-quadrado de Pearson, para verificar se há independência ou alguma relação entre as variáveis que compõem as tabelas.

\section{Resultados e Discussão}

De acordo com o PCDT do Ministério da Saúde (BRASIL, 2013), são incluídos os seguintes códigos da Classificação Internacional de Doenças - 10 (CID-10) para o Lúpus: L93.0 (Lúpus discoide), L93.1 (Lúpus cutâneo subagudo), M32.1 (Lúpus eritematoso disseminado (sistêmico) com comprometimento de outros órgãos e sistemas) e M32.8 (Outras formas de lúpus eritematoso disseminado (sistêmico)). Contudo, neste estudo só foram analisados os pacientes que apresentavam Lúpus Eritematoso Sistêmico (CID-10: M32.1 e M32.8).

O estudo teve início com a observação dos prontuários de pacientes portadores dos CID10 específicos conforme o PCDT para Lúpus (M32.1 e M32.8) cadastrados no Núcleo Regional de Saúde de Vitória da Conquista, acompanhados pelo Componente Especializado da Assistência Farmacêutica, correspondendo a uma amostra total de 110 pacientes.

Entre os 110 pacientes cadastrados, 4,5\% $(\mathrm{n}=5)$ dos indivíduos eram do sexo masculino e 95,5\% ( $n=105)$ do sexo feminino. Marnet e Gonçalves (2016), afirmam que o LES incide muito mais em mulheres do que em homens, em torno de 10 vezes mais. Grossman e Mattson (2014) também consideram maior prevalência no sexo feminino e justificam esse achado devido a um desequilíbrio nos níveis hormonais sexuais, uma vez que os andrógenos sugerem proteção contra o LES, e os estrógenos, ao contrário, favorecem seu desenvolvimento, fazendo com que as respostas imunes dos linfócitos $\mathrm{T}$ auxiliares ganhem mais força e as respostas imunes dos linfócitos $\mathrm{T}$ supressores sejam enfraquecidos, ocasionando assim o desenvolvimento de autoanticorpos. Ao se comparar este estudo com os achados de Silva e Amadei (2001), percebese que a proporção entre homens e mulheres foi o dobro (1:21), esta maior prevalência pode ser explicada devido ao tamanho pequeno da amostra em questão. 
A faixa etária dos pacientes estudados abrangeu entre 19 e 71 anos, o equivalente a uma média de 41,1 anos, estando portando em consenso com Hidalgo-Tenorio (2003) que afirmou que a média de idade dos pacientes lúpicos corresponde a 41,4 anos.

$\mathrm{Na}$ avaliação dos dados demográficos, verificou-se a raça, peso e altura dos pacientes por meio de análise do LME dos pacientes cadastrados. Quanto à raça, foi observada uma maior prevalência da raça parda 59,1\% (n=65) em comparação às outras. Esse achado esteve em conformidade com Oliveira e Almeida (2010) e Bezerra et al (2005) que também obtiveram uma população parda maior de indivíduos com lúpus. Entretanto, há algumas divergências desse achado, uma vez que a raça branca é mais prevalente em um estudo feito por Prado et al (2007) enquanto Conde et al (2009), apontou que o LES é mais frequente em negros. Tal divergência pode ser explicada devido a grande miscigenação presente no país. Na tabela 01 a seguir, podem ser observados os dados sociodemográficos da população lúpica.

Tabela 01: Distribuição segundo sexo, raça e faixa etária de pacientes lúpicos.

\begin{tabular}{l|l|c|c|c}
\hline \multicolumn{2}{c}{} & MASCULINO & FEMININO & TOTAL \\
\hline $\mathbf{N}$ & & 5 & 105 & 110 \\
\hline \multirow{3}{*}{ RAÇA } & Branca & 1 & 29 & 30 \\
\cline { 2 - 5 } & Preta & 1 & 8 & 9 \\
\cline { 2 - 5 } & Parda & 1 & 64 & 65 \\
\cline { 2 - 5 } & Outros & 2 & 4 & 6 \\
\hline \multirow{3}{*}{ FAIXA } & $\mathbf{1 9 - 2 6}$ & 0 & 16 & 16 \\
\cline { 2 - 5 } ETÁRIA & $\mathbf{2 7 - 4 4}$ & 3 & 52 & 55 \\
\cline { 2 - 5 } & $\mathbf{4 5 - 5 6}$ & 1 & 24 & 25 \\
\cline { 2 - 5 } & $\mathbf{5 7 - 7 1}$ & 1 & 13 & 14 \\
\hline
\end{tabular}

Fonte: Dados de Pesquisa (2017).

Foi calculado o Índice de Massa Corporal (IMC) (peso/altura²), baseando-se nos dados coletados, a fim de se fazer a avaliação do estado nutricional da população em estudo, na tentativa de correlacionar os achados com a doença. Tais dados podem ser observados no gráfico 01 , sendo o percentual de pacientes com sobrepeso $45 \%(\mathrm{n}=50)$, um dado que chamou atenção, uma vez que apesar de não se conhecer profundamente a relação entre o estado nutricional e o lúpus, uma dieta equilibrada e balanceada deve ser adotada, a fim de que não se desenvolva outras doenças oportunistas. Klack et al (2012) afirma que uma alimentação que apresente teor energético e proteico, alto teor de vitaminas, minerais e ácidos graxos mono/poli- 
insaturados, são capazes de proteger contra danos tissulares e supressão da atividade inflamatória, podendo ainda ajudar no tratamento dessas comorbidades.

Gráfico 01: IMC pacientes lúpicos cadastrados no NRS.

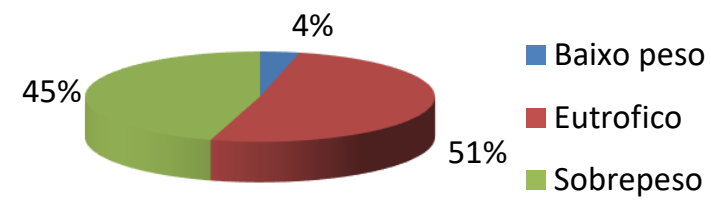

Fonte: Dados de Pesquisa, 2017.

Dos 110 pacientes analisados, pode ser observado na tabela 02 que a Hidroxicloroquina (HID) foi o fármaco mais utilizado no tratamento com $48 \%(\mathrm{n}=83)$, seguido pela Azatioprina (AZA) 32,9\% (n=57), Metotrexato (MTX) 18,5\% (n=32) e pela Ciclosporina (CIC) $0,6 \%(n=1)$.

Tabela 02: Frequência de medicamentos utilizados.

\begin{tabular}{l|c|c}
\hline Medicamentos & $\mathbf{N}$ & $\%$ \\
\hline AZA & 23 & $20,9 \%$ \\
\hline HID & 27 & $24,5 \%$ \\
\hline MTX & 2 & $1,8 \%$ \\
\hline AZA/HID & 28 & $25,5 \%$ \\
\hline AZA/HID/MTX & 5 & $4,5 \%$ \\
\hline AZA/MTX & 1 & $0,9 \%$ \\
\hline CIC/MTX & 1 & $0,9 \%$ \\
\hline HID/MTX & 23 & $20,9 \%$ \\
\hline TOTAL & 110 & $100,0 \%$ \\
\hline
\end{tabular}

Fonte: Dados de Pesquisa (2017).

Foi avaliada a presença de alterações nos exames laboratoriais nos pacientes com LES na tabela 03, por meio de análise de exames solicitados no retorno dos pacientes em acompanhamento no NRS. 
Tabela 03: Correlação entre gênero, presença de alterações hematológicas e tipo de tratamento farmacológico.

\begin{tabular}{|c|c|c|c|c|c|c|c|c|c|c|c|}
\hline \multirow{3}{*}{$\begin{array}{l}\text { PLAQUETAS } \\
\begin{array}{l}\text { Tipo de } \\
\text { tratamento }\end{array} \\
\text { AZA }\end{array}$} & \multirow{3}{*}{$\begin{array}{c}\text { Baixo } \\
00 \% \\
\end{array}$} & \multicolumn{3}{|c|}{ MASCULINO $^{1}$} & \multicolumn{7}{|c|}{ FEMININO $^{2}$} \\
\hline & & \multirow{2}{*}{$\begin{array}{l}\text { Normal } \\
360 \%\end{array}$} & Elevado & \multirow{2}{*}{$\begin{array}{l}\text { Total } \\
360,00 \%\end{array}$} & \multicolumn{2}{|c|}{ Baixo } & \multicolumn{2}{|c|}{ Normal } & Elevado & \multicolumn{2}{|c|}{ Total } \\
\hline & & & $0 \%$ & & 2 & $40 \%$ & 16 & $17,6 \%$ & $0 \quad 0,00 \%$ & 18 & $18,40 \%$ \\
\hline HID & $00 \%$ & $00,00 \%$ & $0 \quad 0 \%$ & $00,00 \%$ & 1 & $20 \%$ & 23 & $25,3 \%$ & $6 \quad 150 \%$ & 25 & $25,50 \%$ \\
\hline MTX & $00 \%$ & $00,00 \%$ & $0 \quad 0 \%$ & $00,00 \%$ & 0 & $0,00 \%$ & 2 & $2,20 \%$ & $60,00 \%$ & 2 & $2,00 \%$ \\
\hline AZA/HID & $00 \%$ & $240 \%$ & $0 \%$ & $240,00 \%$ & 2 & $40 \%$ & 23 & $25,3 \%$ & $0 \quad 0,00 \%$ & 25 & $25,50 \%$ \\
\hline AZA/HID/MTX & $00 \%$ & $00,00 \%$ & $0 \%$ & $00,00 \%$ & 0 & $0,00 \%$ & 4 & $4,40 \%$ & $6 \quad 150 \%$ & 5 & $5,10 \%$ \\
\hline AZA/MTX & $00 \%$ & $0 \longdiv { 0 , 0 0 \% }$ & $0 \%$ & $00,00 \%$ & 0 & $0,00 \%$ & 1 & $1,10 \%$ & $0 \quad 0,00 \%$ & 1 & $1,00 \%$ \\
\hline CIC/MTX & $00 \%$ & $0 \quad 0,00 \%$ & $0 \quad 0 \%$ & $00,00 \%$ & 0 & $0,00 \%$ & 1 & $1,10 \%$ & $6 \quad 0 \quad 0,00 \%$ & 1 & $1,00 \%$ \\
\hline HID/MTX & $00 \%$ & $00,00 \%$ & $0 \quad 0 \%$ & $00,00 \%$ & 0 & $0,00 \%$ & 21 & $23,1 \%$ & $6 \quad 0 \quad 0,00 \%$ & 21 & $21,40 \%$ \\
\hline TOTAL & $00 \%$ & $5100 \%$ & $0 \%$ & $5100 \%$ & 5 & $100 \%$ & 91 & $100 \%$ & $2100 \%$ & 98 & $100,00 \%$ \\
\hline \multicolumn{12}{|c|}{$\begin{array}{l}\text { 1: Não foi possível calcular } \\
\text { 2: Chi-quadrado de Pearson = 13,296 (valor de P: 0,503) }\end{array}$} \\
\hline \multicolumn{2}{|l|}{ LEUCÓCITOS } & \multicolumn{3}{|c|}{ MASCULINO $^{1}$} & \multicolumn{7}{|c|}{ FEMININO $^{2}$} \\
\hline $\begin{array}{l}\text { Tipo de } \\
\text { tratamento }\end{array}$ & Baixo & Normal & Elevado & Total & \multicolumn{2}{|c|}{ Baixo } & \multicolumn{2}{|c|}{ Normal } & Elevado & \multicolumn{2}{|c|}{ Total } \\
\hline AZA & $00 \%$ & $250 \%$ & $1100 \%$ & $360,00 \%$ & 7 & $23,3 \%$ & 10 & $14,9 \%$ & $6 \quad 120 \%$ & 18 & $18,40 \%$ \\
\hline HID & $00 \%$ & $00,00 \%$ & $0 \%$ & $00,00 \%$ & 9 & $30 \%$ & 17 & $25,4 \%$ & $6 \quad 120 \%$ & 27 & $27,60 \%$ \\
\hline MTX & $00 \%$ & $250 \%$ & $0 \%$ & $240,00 \%$ & 0 & $0,00 \%$ & 2 & $3,00 \%$ & $0 \quad 0,00 \%$ & 2 & $2,00 \%$ \\
\hline AZA/HID & $00 \%$ & $0 \quad 0,00 \%$ & $0 \quad 0 \%$ & $00,00 \%$ & 7 & $23,3 \%$ & 16 & $23,9 \%$ & 6. $240 \%$ & 25 & $25,50 \%$ \\
\hline AZA/HID/MTX & $00 \%$ & $0 \quad 0,00 \%$ & $0 \%$ & $00,00 \%$ & 0 & $0,00 \%$ & 5 & $7,50 \%$ & $6 \quad 0 \quad 0,00 \%$ & 5 & $5,10 \%$ \\
\hline AZA/MTX & $00 \%$ & $00,00 \%$ & $0 \%$ & $00,00 \%$ & 0 & $0,00 \%$ & 0 & $0,00 \%$ & $6 \quad 120 \%$ & 1 & $1,00 \%$ \\
\hline CIC/MTX & $00 \%$ & $00,00 \%$ & $0 \%$ & $00,00 \%$ & 0 & $0,00 \%$ & 1 & $1,50 \%$ & $60,00 \%$ & 1 & $1,00 \%$ \\
\hline HID/MTX & $00 \%$ & $00,00 \%$ & $0 \%$ & $00,00 \%$ & 7 & $23,3 \%$ & 16 & $23,9 \%$ & $60,00 \%$ & 23 & $23,50 \%$ \\
\hline TOTAL & $00 \%$ & $4100 \%$ & $1100 \%$ & $5100 \%$ & 30 & $100 \%$ & 67 & $100 \%$ & $5100 \%$ & 102 & $104,10 \%$ \\
\hline \multicolumn{12}{|c|}{$\begin{array}{l}\text { 1: Chi-quadrado de Pearson = 0,833b (valor de P: } 0,361 \text { ) } \\
\text { 2: Chi-quadrado de Pearson = 26,382 (valor de P: 0,023) }\end{array}$} \\
\hline HEMÁCIAS & \multicolumn{4}{|c|}{ MASCULINO $^{1}$} & \multicolumn{7}{|c|}{ FEMININO $^{2}$} \\
\hline $\begin{array}{l}\text { Tipo de } \\
\text { tratamento }\end{array}$ & Baixo & Normal & Elevado & Total & Baix & & No & mal & Elevado & Tota & \\
\hline$\overline{\text { AZA }}$ & $1100 \%$ & $266,7 \%$ & $0 \%$ & $375,00 \%$ & 5 & $45,5 \%$ & 9 & $15,5 \%$ & $60,00 \%$ & 14 & $19,40 \%$ \\
\hline HID & $00 \%$ & $0 \longdiv { 0 , 0 0 \% }$ & $0 \%$ & $00,00 \%$ & 1 & $9,10 \%$ & 12 & $20,7 \%$ & $6 \quad 133,3 \%$ & 14 & $19,40 \%$ \\
\hline MTX & $00 \%$ & $0 \quad 0,00 \%$ & $0 \quad 0 \%$ & $0 \quad 0,00 \%$ & 0 & $0,00 \%$ & 1 & $1,70 \%$ & $6 \quad 0 \quad 0,00 \%$ & 1 & $1,40 \%$ \\
\hline AZA/HID & $00 \%$ & $133,3 \%$ & $0 \quad 0 \%$ & $125,00 \%$ & 1 & $9,10 \%$ & 17 & $29,3 \%$ & $6 \quad 133,3 \%$ & 19 & $26,40 \%$ \\
\hline AZA/HID/MTX & $00 \%$ & $0 \quad 0,00 \%$ & $0 \%$ & $00,00 \%$ & 0 & $0,00 \%$ & 5 & $8,60 \%$ & $6 \quad 0 \quad 0,00 \%$ & 5 & $6,90 \%$ \\
\hline AZA/MTX & $00 \%$ & $00,00 \%$ & $0 \%$ & $00,00 \%$ & 1 & $9,10 \%$ & 0 & $0,00 \%$ & $6 \quad 0 \quad 0,00 \%$ & 1 & $1,40 \%$ \\
\hline CIC/MTX & $00 \%$ & $0 \quad 0,00 \%$ & $0 \%$ & $00,00 \%$ & 0 & $0,00 \%$ & 1 & $1,70 \%$ & $0 \quad 0 \quad 0,00 \%$ & 1 & $1,40 \%$ \\
\hline HID/MTX & $00 \%$ & $0 \quad 0,00 \%$ & $0 \%$ & $00,00 \%$ & 3 & $27,3 \%$ & 13 & $22,4 \%$ & $6 \quad 133,3 \%$ & 17 & $23,60 \%$ \\
\hline TOTAL & $1100 \%$ & $3100 \%$ & $0 \quad 0 \%$ & $480,00 \%$ & 11 & $100 \%$ & 58 & $100 \%$ & $3100 \%$ & 72 & $100,00 \%$ \\
\hline
\end{tabular}

Fonte: Dados da Pesquisa (2017). 
Segundo Costa e Coimbra (2014), a avaliação hematológica indica alterações importantes nos componentes sanguíneos, como baixo número de leucócitos, hemácias, plaquetas. Foram observados distúrbios hematológicos em $21 \%(\mathrm{n}=56)$ dos pacientes dentre os 272 exames hematológicos coletados. Entre os resultados encontrados, 58\% ( $\mathrm{n}=33)$ dos pacientes que estavam em uso de AZA monoterapia ou associada a outro medicamento, manifestaram alterações hematológicas, sendo que em $9 \%(n=3)$ desses pacientes, foi observada a presença de pancitopenia (plaquetas, leucócitos e hemácias diminuídas), confirmando a literatura, uma vez que Neto et al (2008) afirma que a depressão da medula óssea é o efeito tóxico mais comum da AZA, sendo que as citopenias segundo Servioli e colaboradores (2014), que tem ação mielotóxica, pode ser causada pela AZA entre outros medicamentos, o que confirma ainda mais o achado do presente estudo.

Entre os resultados dos pacientes em uso de HID em monoterapia ou associada à AZA e MTX encontrados, $47 \%(n=39)$ dos pacientes apresentaram alterações hematológicas, sendo que a leucopenia esteve presente em $28 \%(n=23)$ dos casos. Quando associado ao MTX, a HID apresentou leucopenia em $8 \%(n=7)$ pacientes, apresentando uma diferença significativa quanto ao reflexo do tipo de tratamento sobre os leucócitos no sexo feminino (teste qui-quadrado, $\mathrm{P}=$ $0,023)$.

Quanto à bicitopenia no uso do MTX, observou-se que dos 32 pacientes analisados, 9\% $(n=3)$ apresentaram tal alteração, divergindo assim de Costa Filho (2014), que em seu estudo, dos 17 pacientes analisados em uso do MTX, 35,2\% (n=6), evidenciando uma ocorrência de bicitopenia acima do encontrado.

Quanto à Ciclosporina (CIC), os achados condizem com a literatura, uma vez que Servioli et al (2014), afirma que a mietotoxicidade não é muito comum no uso deste medicamento, contudo o fato de apenas 1 paciente estar em tratamento com a CIC no estudo em questão, também constitui um fator determinante nos resultados encontrados.

A fim de avaliar os efeitos de toxicidade dos fármacos sobre a função hepática e renal, foram coletados os exames bioquímicos TGO, TGP, fosfatase alcalina, uréia e creatinina, conforme a tabela 04 . 
Tabela 04 : Correlação entre gênero, presença de alterações bioquímicas e tipo de tratamento farmacológico.

\begin{tabular}{|c|c|c|c|c|c|c|c|c|c|c|c|}
\hline \multirow{3}{*}{$\begin{array}{l}\text { TGO E TGP } \\
\text { Tipo de } \\
\text { tratamento } \\
\text { AZA }\end{array}$} & \multicolumn{4}{|c|}{ MASCULINO $^{1}$} & \multicolumn{7}{|c|}{ FEMININO $^{2}$} \\
\hline & Baixo & \multirow{2}{*}{$\begin{array}{l}\text { Normal } \\
375 \%\end{array}$} & \multirow{2}{*}{\begin{tabular}{|c|} 
Elevado \\
$1100 \%$
\end{tabular}} & \multirow{2}{*}{$\begin{array}{l}\text { Total } \\
480 \%\end{array}$} & \multicolumn{2}{|c|}{ Baixo } & \multicolumn{2}{|c|}{ Normal } & Elevado & \multicolumn{2}{|l|}{ Total } \\
\hline & $0 \%$ & & & & 0 & $0 \%$ & 14 & $18,4 \%$ & $26,3 \%$ & 19 & $19,2 \%$ \\
\hline HID & $0 \%$ & $00 \%$ & $00 \%$ & $00 \%$ & 2 & $50 \%$ & 20 & $26,3 \%$ & $15,8 \%$ & 25 & $25,3 \%$ \\
\hline$\overline{\text { MTX }}$ & $0 \%$ & $00 \%$ & $00 \%$ & $00 \%$ & 0 & $0 \%$ & $\overline{1}$ & $1,3 \%$ & $5,3 \%$ & 2 & $2 \%$ \\
\hline AZA/HID & $0 \%$ & $125 \%$ & $00 \%$ & $120 \%$ & 1 & $25 \%$ & 18 & $23,7 \%$ & $21,1 \%$ & 23 & $23,2 \%$ \\
\hline$\overline{\text { AZA/HID/MTX }}$ & $0 \%$ & $00 \%$ & $00 \%$ & $00 \%$ & 0 & $0 \%$ & 3 & $3,9 \%$ & $10,5 \%$ & 5 & $5,1 \%$ \\
\hline AZA/MTX & $0 \%$ & $00 \%$ & $00 \%$ & $00 \%$ & 0 & $0 \%$ & 1 & $1,3 \%$ & $0 \%$ & 1 & $1 \%$ \\
\hline CIC/MTX & $0 \%$ & $00 \%$ & $00 \%$ & $00 \%$ & 0 & $0 \%$ & 1 & $1,3 \%$ & $0 \%$ & 1 & $1 \%$ \\
\hline HID/MTX & $0 \quad 0 \%$ & $00 \%$ & $00 \%$ & $00 \%$ & 1 & $25 \%$ & 18 & $23,7 \%$ & $21,1 \%$ & 23 & $23,2 \%$ \\
\hline TOTAL & $0 \%$ & $4100 \%$ & $1100 \%$ & $5100 \%$ & 4 & $100 \%$ & 76 & $100 \%$ & $19 \quad 100 \%$ & 99 & $100 \%$ \\
\hline \multicolumn{12}{|c|}{ 2: Qui-quadrado de Pearson = 6,448d (valor de P: 0,954) } \\
\hline OSFATASE & \multicolumn{4}{|c|}{ MASCULINO $^{1}$} & \multicolumn{7}{|c|}{ FEMININO $^{2}$} \\
\hline $\begin{array}{l}\text { Tipo de } \\
\text { tratamento }\end{array}$ & Baixo & Normal & Elevado & Total & & aixo & Normal & & Elevado & Tot & \\
\hline AZA & $00 \%$ & $133,3 \%$ & $00 \%$ & $133,3 \%$ & & $0 \%$ & 12 & $17,6 \%$ & $225 \%$ & 14 & $17,9 \%$ \\
\hline HID & $00 \%$ & $\begin{array}{ll}0 & 0 \% \\
\end{array}$ & $00 \%$ & $00 \%$ & & $100 \%$ & 16 & $23,5 \%$ & $225 \%$ & 20 & $25,6 \%$ \\
\hline MTX & $00 \%$ & $\begin{array}{ll}0 & 0 \% \\
\end{array}$ & $00 \%$ & $00 \%$ & & $0 \%$ & 2 & $2,9 \%$ & $0 \quad 0 \%$ & 2 & $2,6 \%$ \\
\hline AZA/HID & $00 \%$ & $266,7 \%$ & $00 \%$ & $266,7 \%$ & & $0 \%$ & 15 & $22,1 \%$ & $225 \%$ & 17 & $21,8 \%$ \\
\hline AZA/HID/MTX & $00 \%$ & $\begin{array}{ll}0 & 0 \% \\
\end{array}$ & $00 \%$ & $00 \%$ & & $0 \%$ & 3 & $4,4 \%$ & $\begin{array}{ll}0 & 0 \% \\
\end{array}$ & 3 & $3,8 \%$ \\
\hline AZA/MTX & $00 \%$ & $\begin{array}{ll}0 & 0 \% \\
\end{array}$ & $00 \%$ & $00 \%$ & & $0 \%$ & 1 & $1,5 \%$ & $0 \quad 0 \%$ & 1 & $1,3 \%$ \\
\hline CIC/MTX & $00 \%$ & $\begin{array}{ll}0 & 0 \% \\
\end{array}$ & $00 \%$ & $00 \%$ & & $0 \%$ & 1 & $1,5 \%$ & $\begin{array}{ll}0 & 0 \% \\
\end{array}$ & 1 & $1,3 \%$ \\
\hline HID/MTX & $00 \%$ & $\begin{array}{ll}0 & 0 \% \\
\end{array}$ & $00 \%$ & $00 \%$ & & $0 \%$ & 18 & $26,5 \%$ & $225 \%$ & 20 & $25,6 \%$ \\
\hline TOTAL & $00 \%$ & $3100 \%$ & $00 \%$ & $3100 \%$ & & $100 \%$ & 68 & $100 \%$ & $8 \quad 100 \%$ & 78 & $100 \%$ \\
\hline \multicolumn{12}{|c|}{$\begin{array}{l}\text { 1: Não foi possível calcular } \\
\text { 2: Qui-quadrado de Pearson }=7,054 \mathrm{c} \text { (valor de P: } 0,933 \text { ) }\end{array}$} \\
\hline $\begin{array}{c}\text { URÉIA E } \\
\text { CREATININA }\end{array}$ & \multicolumn{4}{|c|}{ MASCULINO $^{1}$} & \multicolumn{7}{|c|}{ FEMININO $^{2}$} \\
\hline $\begin{array}{l}\text { Tipo de } \\
\text { tratamento }\end{array}$ & Baixo & Normal & Elevado & Total & Bair & & Norr & mal & Elevado & Total & \\
\hline AZA & $00 \%$ & $250 \%$ & $250 \%$ & $444,4 \%$ & 3 & $30 \%$ & 13 & $18,1 \%$ & $6228,6 \%$ & 18 & $20,2 \%$ \\
\hline HID & $00 \%$ & $00 \%$ & $00 \%$ & $00 \%$ & 2 & $20 \%$ & 17 & $23,6 \%$ & $6 \quad 1 \quad 14,3 \%$ & 20 & $22,5 \%$ \\
\hline MTX & $00 \%$ & $250 \%$ & $00 \%$ & $222,2 \%$ & 0 & $0 \%$ & 1 & $1,4 \%$ & $114,3 \%$ & 2 & $2,2 \%$ \\
\hline AZA/HID & $1100 \%$ & $00 \%$ & $250 \%$ & $333,3 \%$ & 1 & $10 \%$ & 18 & $25 \%$ & $342,9 \%$ & 22 & $24,7 \%$ \\
\hline AZA/HID/MTX & $00 \%$ & $00 \%$ & $00 \%$ & $00 \%$ & 0 & $0 \%$ & 4 & $5,6 \%$ & $00 \%$ & 4 & $4,5 \%$ \\
\hline AZA/MTX & $00 \%$ & $00 \%$ & $00 \%$ & $00 \%$ & 1 & $10 \%$ & 0 & $0 \%$ & $00 \%$ & 1 & $1,1 \%$ \\
\hline CIC/MTX & $00 \%$ & $00 \%$ & $00 \%$ & $00 \%$ & 0 & $0 \%$ & 1 & $1,4 \%$ & $00 \%$ & 1 & $1,1 \%$ \\
\hline HID/MTX & $00 \%$ & $00 \%$ & $00 \%$ & $00 \%$ & 3 & $30 \%$ & 18 & $25 \%$ & $00 \%$ & 21 & $23,6 \%$ \\
\hline TOTAL & $1100 \%$ & $4100 \%$ & $4100 \%$ & $9100 \%$ & 10 & $100 \%$ & 672 & $100 \%$ & $7100 \%$ & 89 & $100,0 \%$ \\
\hline $\begin{array}{l}\text { 1: Qui-quadrado de } \\
\text { 2: Qui-quadrado de }\end{array}$ & $\begin{array}{l}\text { earson }=2 \\
\text { earson }=1\end{array}$ & $\begin{array}{l}2,917 \mathrm{~b}(\mathrm{va} \\
18,910 \mathrm{c}(\mathrm{v}\end{array}$ & $\begin{array}{l}\text { or de P: } 0,2 \\
\text { lor de P: } 0\end{array}$ & & & & & & & & \\
\hline
\end{tabular}

Fonte: Dados da Pesquisa (2017). 
Entre os resultados dos pacientes do sexo masculino, 20\% $(n=1)$ apresentou TGO e TGP elevado, sendo este no uso da AZA. Já entre os pacientes do sexo feminino, percebeu-se que das 27 das mulheres com TGO, TGP e/ou fosfatase alcalina elevados, 56\% $(n=15)$ faziam uso da AZA, sendo que desses, 30\% (n=8) estavam associados à HID, seguidos de outros 7\% (n=2) no uso do MTX associado à HID e AZA. O dado revelado concorda com a literatura, por meio da qual Deleve et al (1996), destaca a AZA como um medicamento que tem como órgão alvo de ataque o fígado, por estar associado ao primeiro passo da biotransformação, na qual há depleção dos níveis do detoxificador glutationa e aumento dos níveis de 6metilmercaptopurina, que está relacionada com a hepatotoxicidade. A HID também apresenta com potencial efeito tóxico ao fígado, devido a frequente aumento da fosfatase, embora não tenha sido encontrada uma diferença estatisticamente significativa (teste qui-quadrado, $\mathrm{P}=0,171$ / P: 0,954). Quanto à avaliação da função renal dos pacientes com lúpus, por meio da uréia ou creatinina elevados, percebeu-se que $11 \% \quad(\mathrm{n}=11)$ dos casos encontrados possuem comprometimento renal. Esse achado condiz com a literatura, uma vez que Kobayashi (2013), revela que é muito frequente o acometimento renal nos pacientes lúpicos, apesar de não ter sido encontrada uma diferença estatisticamente significativa.

Na tabela 05 , foi avaliada a resposta inflamatória, foram observados o VHS e o PCR, sendo que os resultados confirmam a quantidade de inflamação que o paciente com LES apresenta.

Tabela 05: Correlação entre gênero, presença de alterações imunológicas e tipo de tratamento farmacológico.

\begin{tabular}{|c|c|c|c|c|c|c|c|c|c|c|c|}
\hline \multirow{3}{*}{$\begin{array}{l}\text { VHS E PCR } \\
\text { Tipo de tratamento } \\
\text { AZA }\end{array}$} & \multicolumn{4}{|c|}{ MASCULINO ${ }^{1}$} & \multicolumn{7}{|c|}{ FEMININO $^{2}$} \\
\hline & Baixo & \multirow{2}{*}{$\begin{array}{l}\text { Normal } \\
150 \%\end{array}$} & \multirow{2}{*}{ 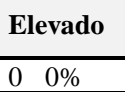 } & Total & Baixo & \multicolumn{2}{|c|}{ Normal } & \multicolumn{2}{|c|}{ Elevado } & \multicolumn{2}{|c|}{ Total } \\
\hline & $0 \quad 0 \%$ & & & $133,3 \%$ & $00 \%$ & 7 & $15,6 \%$ & 5 & $12,8 \%$ & 12 & $13,8 \%$ \\
\hline HID & $0 \quad 0 \%$ & $00 \%$ & $0 \quad 0 \%$ & $0 \quad 0 \%$ & $00 \%$ & 14 & $31,1 \%$ & 11 & $28,2 \%$ & 25 & $28,7 \%$ \\
\hline MTX & $0 \quad 0 \%$ & $00 \%$ & $0 \quad 0 \%$ & $0 \quad 0 \%$ & $0 \quad 0 \%$ & 1 & $2,2 \%$ & 1 & $2,6 \%$ & 2 & $2,3 \%$ \\
\hline AZA/HID & $0 \quad 0 \%$ & $150 \%$ & $1100 \%$ & $266,7 \%$ & $0 \quad 0 \%$ & 12 & $26,7 \%$ & 8 & $20,5 \%$ & 20 & $23 \%$ \\
\hline AZA/HID/MTX & $0 \quad 0 \%$ & $00 \%$ & $0 \quad 0 \%$ & $0 \quad 0 \%$ & $0 \quad 0 \%$ & 2 & $4,4 \%$ & 3 & $7,7 \%$ & 5 & $5,7 \%$ \\
\hline AZA/MTX & $0 \quad 0 \%$ & $00 \%$ & $0 \quad 0 \%$ & $0 \quad 0 \%$ & $0 \quad 0 \%$ & 0 & $0,0 \%$ & 1 & $2,6 \%$ & 1 & $1,1 \%$ \\
\hline CIC/MTX & $0 \quad 0 \%$ & $00 \%$ & $0 \quad 0 \%$ & $0 \quad 0 \%$ & $0 \quad 0 \%$ & 1 & $2,2 \%$ & 0 & $0 \%$ & 1 & $1,1 \%$ \\
\hline HID/MTX & $0 \quad 0 \%$ & $00 \%$ & $0 \quad 0 \%$ & $0 \quad 0 \%$ & $3100 \%$ & 8 & $17,8 \%$ & 10 & $25,6 \%$ & 21 & $24,1 \%$ \\
\hline TOTAL & $0 \quad 0 \%$ & $2100 \%$ & $1100 \%$ & $3100 \%$ & $3100 \%$ & 45 & $100 \%$ & 39 & $100 \%$ & 87 & $100 \%$ \\
\hline $\begin{array}{l}\text { 1: Qui-quadrado de } \mathrm{P} \\
\text { 2: Qui-quadrado de } \mathrm{P}\end{array}$ & on $=1$ & $\begin{array}{l}\text { Ob (valor } \\
06 \mathrm{~d} \text { (valc }\end{array}$ & $\begin{array}{l}P: 0,386) \\
\text { P: } 0,503\end{array}$ & & & & & & & & \\
\hline
\end{tabular}

Fonte: Dados da Pesquisa (2017). 
Apesar de não ter apresentado significância estatística (teste qui-quadrado, $\mathrm{P}=0,386$ / $\mathrm{P}=0,503)$, pode-se perceber a ocorrência de PCR e VHS elevados em 44\% (n=40) dos pacientes, o que sugere uma possível inefetividade do tratamento. A HID foi a que mais frequentemente se elevou com $37 \%(n=33)$, todavia, não foi encontrada na literatura relação VHS e PCR elevados com a HID.

Tabela 6: Medicamentos prescritos para tratamento do LES de acordo aos prescritores.

\begin{tabular}{l|c|c|c|c|c}
\hline $\begin{array}{l}\text { TIPO DE } \\
\text { TRATAMENTO }\end{array}$ & N & PRESCRITOR A & PRESCRITOR B & $\begin{array}{c}\text { PRESCRITOR } \\
\text { C }\end{array}$ & OUTROS \\
\hline AZA & 23 & 3 & 3 & 3 & 14 \\
\hline HID & 27 & 0 & 10 & 9 & 8 \\
\hline MTX & 2 & 0 & 1 & 1 & 0 \\
\hline AZA/HID & 28 & 4 & 4 & 13 & 7 \\
\hline AZA/HID/MTX & 5 & 1 & 2 & 0 & 2 \\
\hline AZA/MTX & 1 & 0 & 1 & 0 & 0 \\
\hline CIC/MTX & 1 & 0 & 0 & 0 & 1 \\
\hline HID/MTX & 23 & 3 & 4 & 12 & 4 \\
\hline TOTAL & 110 & 11 & 25 & 38 & 36 \\
\hline
\end{tabular}

Fonte: Dados de Pesquisa (2017).

Nota-se na tabela 06, que há algumas variações nos medicamentos prescritos, de modo que os medicamentos que mais prescritos são Azatioprina e Hidroxicloroquina, em monoterapia ou associados entre si. Com isso foi possível observar a prevalência não só do medicamento utilizado, como também uma possível tendência de escolha do prescritor, levantando a possibilidade de que o tratamento medicamentoso se repete devido a padronização definida individualmente pelo prescritor no momento da instituição da farmacoterapia.

O Núcleo Regional de Saúde, onde foi realizado o estudo é localizado em Vitória da Conquista, no interior da Bahia, sendo responsável pelo atendimento a pacientes da cidade e regiões circunvizinhas. A partir disso, foi feito um levantamento da quantidade de pacientes lúpicos que são atendidos no Núcleo, de acordo a cada cidade beneficiada pelo mesmo, podendo ser observado na figura 05 a seguir, onde se constatou que o maior número em questão atendidos, se encontram em Vitória da Conquista, provavelmente devido ao tamanho da população, bem como maior facilidade de acesso por localização, conforme o quadro 01 a seguir. 
Gráfico 01: Representação da quantidade de pacientes lúpicos atendidos por região, no Núcleo Regional de Saúde de Vitória da Conquista, 2017.

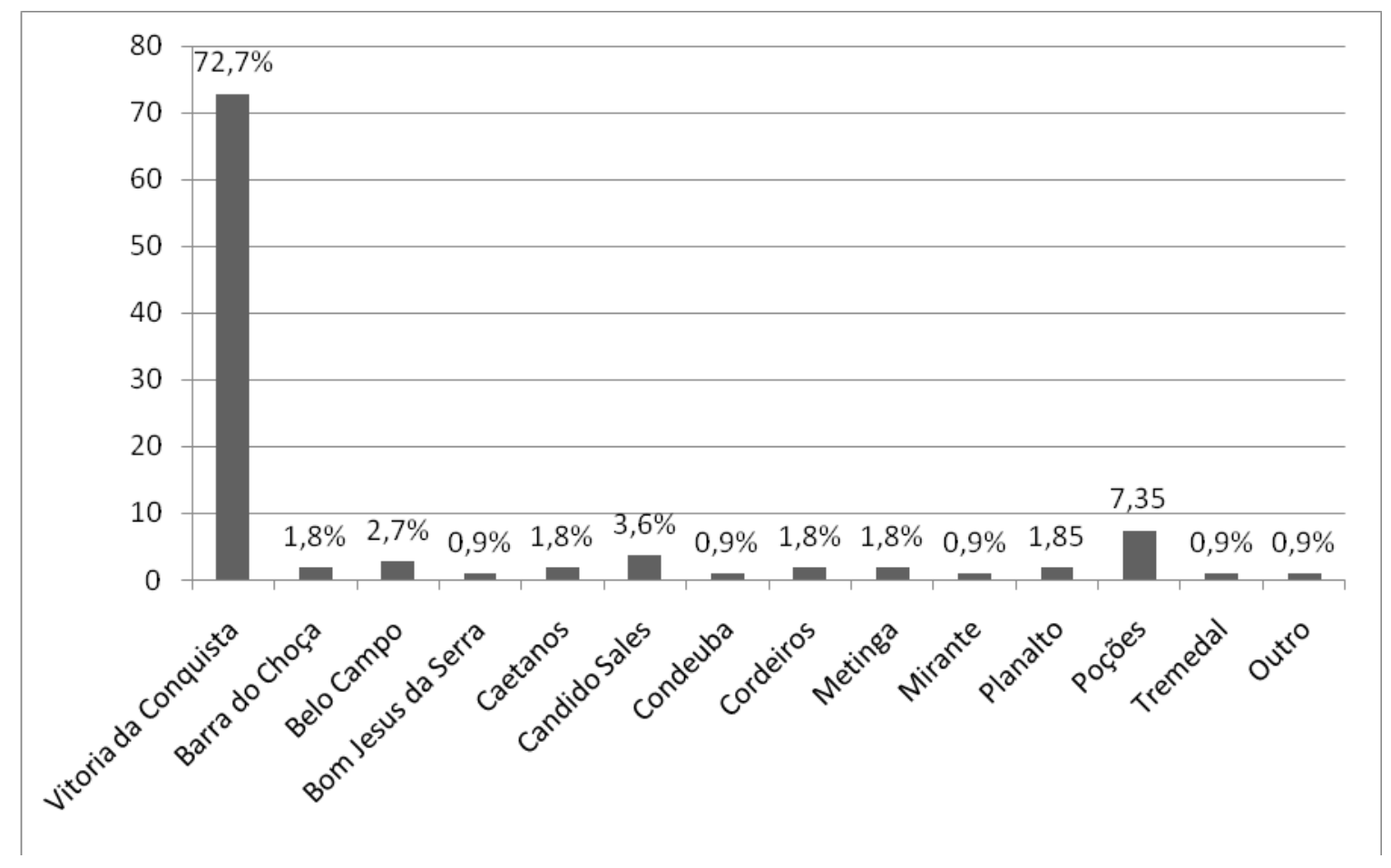

Fonte: Dados de Pesquisa (2017).

\section{Conclusão}

O estudo pôde revelar o perfil dos pacientes portadores de LES, no que se refere à aspectos sociodemográficos e clínicos, podendo através dos resultados obtidos aumentar as informações referentes à doença. Na população em questão, os pacientes apresentaram um perfil condizente com o esperado pela literatura, sendo na sua maioria do sexo feminino, pardos, adultos jovens, com IMC normal.

Embora a Azatioprina, constitua um fármaco efetivo no tratamento do LES, percebeuse uma frequência maior associada a distúrbios hematológicos, renais e hepáticos, paralelo à Hidroxicloroquina com provas de atividade inflamatória elevada. Simultaneamente, foi possível perceber que alguns prescritores prescrevem sempre os mesmos medicamentos, de forma padronizada. Esse fato pode estar associado ao aparecimento de tais distúrbios em alguns 
pacientes, visto que a farmacoterapia deve ser ajustada de acordo às necessidades individuais de cada paciente.

Esses achados revelam, portanto, que a Azatioprina deve ser monitorada, uma vez que tem apresentado risco de danos à medula óssea, por meio dos distúrbios hematológicos. Paralelo a isso, a Hidroxicloroquina constitui um indicador de inefetividade para o paciente, visto que apresentou valores elevados quanto ao PCR e VHS, por ter aparecido elevado com frequência nos marcadores de prova de atividade inflamatória. Com isso, percebe-se a importância de se fazer um monitoramento farmacoterapêutico do paciente, esperando-se maior segurança no tratamento instituído, a fim de se obter uma melhora no quadro clínico e consequentemente na qualidade de vida do mesmo.

\section{Referências}

ABBAS. A. K.; LICHTMAN. A. H.; PILLAI. S. Imunologia: celular e molecular. 6. ed. Rio de Janeiro: Elsevier, 2008. 564p.

OLIVEIRA, Ana Paula de, ALMEIDA, Thaíse Sousa Brito de. Perfil dos Portadores de Lúpus Eritematoso Sistêmico Atendidos no Ambulatório De Reumatologia do Hospital de Base do Distrito Federal em 2010. 48 f. Taguatinga-DF, Universidade Católica de Brasília. Taguatinga, 2010.

AYACHE, Danusa Céspedes Guizzo; COSTA, Izaías Pereira Da. Alterações da Personalidade no Lúpus Eritematoso Sistêmico. Revista Brasileira de Reumatologia, Campo Grande- MS, v. 45, n. 5, p. 313-318, Set./Out. 2005.

BRASIL, Ministério da Saúde. Aprova o Protocolo Clínico e Diretrizes Terapêuticas do Lúpus Eritematoso Sistêmico. Portaria nº 100, de 7 de Fevereiro de 2013.

BRASIL, Ministério da Saúde. Dispõe sobre as regras de financiamento e execução do Componente Especializado da Assistência Farmacêutica no âmbito do Sistema Único de Saúde (SUS). Portaria nº 1.554, de 30 de Jul. de 2013.

BEZERRA et al. Lúpus Eritematoso Sistêmico (LES): Perfil Clínico-Laboratorial dos Pacientes do Hospital Universitário Onofre Lopes (UFRN-Natal/Brasil) e Índice de Dano nos Pacientes com Diagnóstico Recente (*). Rev Bras Reumatol, v. 45, n. 6, p. 339-342, nov./dez., 2005.

CONDE, S. R. et al. Estudo clínico-epidemiológico de pacientes com lupus eritematoso sistêmico, em uma população da amazônia oriental. Belém/Pará/Brasil, 2009. 
COSTA FILHO, José Djandir. Alterações na medula óssea de pacientes com doenças autoimunes sob tratamento com imunossupressores. 2014. 77 f. Dissertação (Mestrado em Patologia) - Faculdade de Medicina, Universidade Federal do Ceará, Fortaleza, 2014.

COSTA, Luciana Meira Da; COIMBRA, Claudia Cristina Batista Evangelista. Lúpus eritematoso sistêmico: incidência e tratamento em mulheres. Revista uningá review, Maringá, v. 20, n. 1, p. 81-86, set. 2014.

GALINDO, Cícera V. F.; VEIGA, Renata K. A. Características Clínicas e Diagnóstico do Lúpus Eritematoso: Uma Revisão. Revista Eletrônica de Farmácia REF. ISSN 1808-0804 Vol. VII (4), 46 - 58, 2010.

GROSSMAN, Sheila C.; PORTH, Carol Mattson (2014). PorthFisiopatologia; [Tradução de Carlos Henrique de Araújo Cosendey, MaizaRitomy Ide, Mariângela Vidal Sampaio Fernandes e Sylvia Werdmüller von Elgg Roberto]. - 9. Ed. - Rio de Janeiro: Guanabara Koogan, 2016. p. 1531-1533.

HIDALGO-TENORIO, C. et al. Urinary tract infections and lupus erythematosus. Ann Rheum Dis, Spain, v. 63, p. 431-437, jul. 2003.

HOCHBERG, M C. Atualização do American CollegeofRheumatology critérios revistos para a classificação do lúpus eritematoso sistêmico. Artrite Rheum., 1997.

KLACK, Karin; BONFA, Eloisa; NETO, Eduardo Ferreira Borba. Dieta e aspectos nutricionais no lúpus eritematoso sistêmico. Revista brasileira de reumatologia, São paulo, v. 52, n. 3, jun./set. 2014.

KOBAYASHI, Carla Dinamérica. Incidência e perfil clínico da nefrite lúpica nos pacientes submetidos à biópsia renal entre 2007 e $2012 \mathrm{em}$ um hospital de referência em nefrologia no Estado da Bahia. Salvador: 2012. 30 p.

KoboToolbox. KoBoToolbox at the Harvard Humanitarian Initiative. 2016; http://www.kobotoolbox.org/. Disponível em: http://www.kobotoolbox.org/. Acessado em 07 set., 2017.

MARNET, Érica Buday De Oliveira; GONÇALVES, Marcia. Lúpus Eritematoso Sistêmico e sua Associação com Doenças Psiquiátricas: Revisão da Literatura. Psychiatry on line Brasil, Taubaté, v. 21, n. 7, jul. 2016.

OLIVEIRA, Ana Paula de, ALMEIDA, Thaíse Sousa Brito de. Perfil dos Portadores de Lúpus Eritematoso Sistêmico Atendidos no Ambulatório De Reumatologia do Hospital de Base do Distrito Federal em 2010. 48 f. Taguatinga-DF, Universidade Católica de Brasília. Taguatinga, 2010.

PRADO et al. Perfil clínico-laboratorial de pacientes do Ambulatório de Lupus Eritematoso Sistêmico do Hospital São Lucas da PUCRS. Scientia Medica, Porto Alegre, v. 17, n. 3, p. 168-170, jul./set. 2007.

PETRI, M. et al. Derivação e Validação de Lupus Sistêmico: Colaboração Internacional Clínica para Critérios de Classificação para Lúpus Eritematoso Sistêmico. ArthritisRheum, USA, v. 64, n. 8, ago. 2012. 
SATO, E. I. et al. Consenso Brasileiro para o Tratamento do Lúpus Eritematoso Sistêmico (LES). Revista Brasileira de Reumatologia, São Paulo, v. 42, n. 6, p. 362-370, Nov./Dez. 2002.

SERVIOLI et al. Citopenias hematológicas en enfermedades autoinmunes sistémicas Blood cytopenias in systemic autoimmune conditions. Arch Med Interna 2014; 36(3):101-109. Uruguai, 2014.

SOUSA, Kay Anne Gomes; SILVA, Vanuza Soares Da; AnetteKelseiPartata. A Importância do Profissional Farmacêutico no aconselhamento ao portador de Lúpus Eritematoso Sistêmico. revista científica do itpac, Tocantins, v. 4, n. 1, Nov./Dez. 2002.

VIANNA, Rodrigo; SIMÕES, Manuel Jesus; INFORZATO, Heraldo C. Borges. Lúpus Eritematoso Sistêmico. Revista CecilianaJun 2 (1): 1-3, 2010 ISSN 2175-7224 - 2009/2010Universidade Santa Cecília.

\section{Como citar este artigo (Formato ABNT):}

PRADO, Diane de M.; AMARAL, Bruno A.; DUARTE, Stênio F.P.; DO VALE, Ademir E.; SILVA, Matheus L.; ROCHA, Arlene R.; SOUSA, Vitória M. Perfil dos Pacientes portadores de Lúpus Eritematoso Sistêmico atendidos pelo Componente Especializado da Assistência Farmacêutica em uma Regional de Saúde. Id on Line Revista Multidisciplinar e de Psicologia, 2017, vol.11, n.38, p.808823. ISSN: 1981-1179.

Recebido: 08.11.2017

Aceito: 10.11 .2017 